\title{
Significant lethality following liver resection in A20 heterozygous knockout mice uncovers a key role for A20 in liver regeneration
}

\author{
P Studer ${ }^{1,2}$, CG da Silva ${ }^{1}$, JM Revuelta Cervantes ${ }^{1}$, A Mele ${ }^{1}$, E Csizmadia ${ }^{1}$, JJ Siracuse ${ }^{1}$, SM Damrauer ${ }^{1}$, CR Peterson ${ }^{1}$, D Candinas ${ }^{2}$, \\ DM Stroka ${ }^{2}, \mathrm{~A} \mathrm{Ma}^{3}, \mathrm{M}$ Bhasin $^{4}$ and C Ferran ${ }^{*, 1}$
}

Hepatic expression of A20, including in hepatocytes, increases in response to injury, inflammation and resection. This increase likely serves a hepatoprotective purpose. The characteristic unfettered liver inflammation and necrosis in A20 knockout mice established physiologic upregulation of $\mathrm{A20}$ as integral to the anti-inflammatory and anti-apoptotic armamentarium of hepatocytes. However, the implication of physiologic upregulation of A20 in modulating hepatocytes' proliferative responses following liver resection remains controversial. To resolve the impact of $A 20$ on hepatocyte proliferation and the liver's regenerative capacity, we examined whether decreased A20 expression, as in A20 heterozygous knockout mice, affects outcome following two-third partial hepatectomy. A20 heterozygous mice do not demonstrate a striking liver phenotype, indicating that their A20 expression levels are still sufficient to contain inflammation and cell death at baseline. However, usually benign partial hepatectomy provoked a staggering lethality $(>40 \%)$ in these mice, uncovering an unsuspected phenotype. Heightened lethality in A20 heterozygous mice following partial hepatectomy resulted from impaired hepatocyte proliferation due to heightened levels of cyclin-dependent kinase inhibitor, p21, and deficient upregulation of cyclins D1, E and A, in the context of worsened liver steatosis. A20 heterozygous knockout minimally affected baseline liver transcriptome, mostly circadian rhythm genes. Nevertheless, this caused differential expression of $>1000$ genes post hepatectomy, hindering lipid metabolism, bile acid biosynthesis, insulin signaling and cell cycle, all critical cellular processes for liver regeneration. These results demonstrate that mere reduction of $\mathrm{A20}$ levels causes worse outcome post hepatectomy than full knockout of bona fide liver pro-regenerative players such as IL-6, clearly ascertaining A20's primordial role in enabling liver regeneration. Clinical implications of these data are of utmost importance as they caution safety of extensive hepatectomy for donation or tumor in carriers of A20/TNFAIP3 single nucleotide polymorphisms alleles that decrease A20 expression or function, and prompt the development of A20-based liver proregenerative therapies.

Cell Death and Differentiation (2015) 22, 2068-2077; doi:10.1038/cdd.2015.52; published online 15 May 2015

Humans and mice restore their liver mass within 2 weeks of surgical, viral or toxic parenchymal loss via compensatory regrowth, referred to as liver regeneration (LR). ${ }^{1,2}$ Adequate LR is contingent upon maintaining a minimum healthy residual liver mass without which hepatocyte proliferation is stunned, resulting in hepatic failure. In liver transplantation (mainly living donor liver transplantation), 'small-for-size' syndrome illustrates this outcome. ${ }^{3}$ Incremental $78 \%$ and $87 \%$ hepatectomy in mice reproduces this syndrome, causing $50 \%$ and $100 \%$ lethality, respectively. ${ }^{4}$ Despite considerable knowledge characterizing the molecular basis of LR, better understanding of this process's shortcomings in the face of extensive resection and/or damage is required for uncovering therapies to overcome defective regeneration. These discoveries would revolutionize clinical management of large liver tumors and of liver transplantation. ${ }^{5,6}$

Our laboratory contributed novel leads to such therapies by exposing the dramatic survival advantage afforded by hepatic overexpression of NF- $\kappa$ B-inhibitory and ubiquitin-editing protein, $A 20,7,8$ in mouse models of severe toxic or ischemic liver injuries, ${ }^{9,10}$ and of extended (78\%) and radical (87\%) hepatectomy. ${ }^{4}$ A20 confers this advantage by (i) limiting

\footnotetext{
${ }^{1}$ Division of Vascular Surgery, Center for Vascular biology Research and the Transplant Institute, Department of Surgery, Beth Israel Deaconess Medical Center, Harvard Medical School, Boston, MA, USA; ${ }^{2}$ Department of Visceral Surgery and Medicine, University Hospital Bern, Bern, Switzerland; ${ }^{3}$ Division of Interdisciplinary Medicine and Biotechnology, Bioinformatics core, Beth Israel Deaconess Medical Center, Harvard Medical School, Boston, MA, USA and ${ }^{4}$ Division of Gastroenterology, Department of Medicine, University of California in San Francisco, San Fransisco, CA, USA

*Corresponding author: C Ferran, Beth Israel Deaconess Medical Center, 99 Brookline Avenue, Research North \#370 F/G, Boston, MA 02215 , USA. Tel: +1 617 667 0440; Fax: +1 617 6670445; E-mail: cferran@ bidmc.harvard.edu

Abbreviations: ARNTL, aryl hydrocarbon receptor nuclear translocator like; BMAL1, brain and muscle ARNTL; Cyp7a1, cholesterol 7 alpha hydroxylase; CLOCK, circadian locomotor output cycles kaput; CR, circadian rhythm; CRTF, circadian rhythm transcription factors; CCND1, cyclin D1; CDKI, cyclin-dependent kinase inhibitor; DBP, D site of albumin promoter binding protein; FA, fatty acids; GO, gene ontology; HGF, hepatocyte growth factor; HET, heterozygous; INSIG2, insulin induced regulator of SREBP1c; KO, knockout; LR, liver regeneration; NPAS2, neuronal PAS domain-containing protein 2; PCNA, proliferating cell nuclear antigen; PH, partial hepatectomy; PPAR $\alpha$, peroxisome proliferator activated receptor-alpha; PDK4, pyrruvate dehydrogenase kinase isozyme 4; RGS16, regulator of G-coupled receptor signaling 16; STAT3, signal transducer and activator of transcription-3; SNPs, single nucleotide polymorphisms; TGF $\alpha$, transforming growth factor alpha

Received 29.9.14; revised 24.2.15; accepted 13.3.15; Edited by P Salomoni; published online 15.5.15
} 
inflammation through inhibition of NF- $\kappa \mathrm{B}$ activation, (ii) protecting hepatocytes from apoptosis, ${ }^{9}$ (iii) reducing oxidative stress by increasing peroxisome proliferator-activated receptoralpha (PPARa) expression, ${ }^{10}$ (iv) promoting hepatocyte proliferation by decreasing cyclin-dependent kinase inhibitor (CDKI) p21, enhancing interleukin-6/signal transducer and activator of transcription-3 (IL-6/STAT3) regenerative signals, and (v) optimizing energy production by improving fatty acid (FA) metabolism. ${ }^{4,11,12}$ A20 homozygous $\mathrm{KO}$ mice are born cachectic, and die within 3-6 weeks of birth from systemic inflammation, predominantly in the liver. ${ }^{13}$ This highlights A20's prominence in the liver's physiologic anti-inflammatory and anti-apoptotic defense mechanisms. Significant upregulation of $\mathrm{A} 20$ in humans and mice livers following hepatectomy suggests that $\mathrm{A} 20$ is also an active player in the liver's physiologic regenerative response. ${ }^{1,4,9,12}$ We explored this hypothesis by evaluating LR following two-third partial hepatectomy $(\mathrm{PH})$ in female $\mathrm{A} 20$ heterozygous knockout (HET) mice that show no pathological phenotype at baseline, including in the liver. This procedure is impossible in shortlived cachectic A20 KO mice. ${ }^{13}$

\section{Results}

A20 knockdown provokes lethality by increasing liver damage and impairing hepatocyte proliferation following PH. Two-third PH, a non-lethal procedure in wild-type (WT) mice, resulted in a staggering $42 \%$ lethality in HET mice within 7 days (d) of surgery (Figure 1a). Lethality in HET mice associated with greater liver damage, as evidenced by significantly higher plasma levels of alanine transaminase, aspartate transaminase and total bilirubin 1-2 d post $\mathrm{PH}$ (Figures $1 b-d)$, and on morphometry by increased liver steatosis (2 d), necrosis (4 d) and hemorrhage (7d) in HET versus WT livers (Figure 1e). Lethality in HET mice also correlated with decreased hepatocyte proliferation, as evidenced by nearly absent $\mathrm{Ki} 67^{+}$hepatocytes/high power field in HET livers 2 d post PH. Hepatocyte proliferation occurred in HET mice that survived the procedure, but was delayed (4 d) and never as robust as in WT (Figure 1f). By western blot analysis, we also checked for hepatic expression of proliferating cell nuclear antigen (PCNA), an auxiliary protein to DNA polymerase delta that is required for cell proliferation. PCNA levels in WT livers increased $1 \mathrm{~d}$, peaked $2 \mathrm{~d}$ and tapered down $4 \mathrm{~d}$ after $\mathrm{PH}$, as expected. ${ }^{14} \mathrm{PCNA}$ levels also increased in livers of HET mice $1 \mathrm{~d}$ post $\mathrm{PH}$, but became undetectable by 2 d post $\mathrm{PH}$, marking aborted LR. PCNA expression increased again in HET mice that survived $\mathrm{PH}$ but was delayed (4 and $7 \mathrm{~d}$ post $\mathrm{PH}$; Figure 1g).

A20 is part of the liver regenerative response. ${ }^{1,4}$ Upregulation of A20 mRNA and protein in HET livers following $\mathrm{PH}$ was insufficient, that is, transient (limited to $1 \mathrm{~d}$ post $\mathrm{PH}$ ) and significantly lower than in WT livers (Figures $1 \mathrm{~h}$ and i). These results link inadequate upregulation of A20 in HET livers post $\mathrm{PH}$ (both temporal and quantitative) to their defective LR. A20 protein levels were moderately higher in livers of A20 HET mice at baseline, agreeing with increased activation in these livers of the transcriptional regulator of $A 20, N F-k B .^{9,15,16}$
A20 HET livers demonstrate heightened intra-hepatic levels of TNF and IL- 6 but no increase in hepatocyte apoptosis following $\mathrm{PH}$. $\mathrm{PH}$ increases circulating and intrahepatic TNF and IL-6 levels to prime entry of hepatocytes into cell cycle. ${ }^{2}$ Exaggerated production of these priming, yet pro-inflammatory, cytokines stalls hepatocyte proliferation, hampering LR. ${ }^{17}$ A20 heterozygocity did not affect basal circulating levels of TNF and IL-6 nor early (first $24 \mathrm{~h}$ ) TNF upregulation post $\mathrm{PH}$, and only caused moderately higher circulating IL- 6 levels $6 \mathrm{~h}$ post PH in HET versus WT mice (Figures $2 \mathrm{a}$ and $\mathrm{b}$ ). This result is in keeping with the absence of an overt hyperactivation of the innate and adaptive immune system in A20 HET mice, which contrasts with what observed in $\mathrm{A} 20 \mathrm{KO}$ mice. $^{13}$ However, we noted significantly higher intrahepatic TNF (10-20-fold) and IL-6 (10-15-fold) mRNA levels in HET versus WT livers 2 d post PH (Figures 2c and d). This correlated with significantly higher intra-hepatic inflammation, as gauged by nuclear expression of the NF- $k \mathrm{~B}$ subunit p65 in the liver, mostly in hepatocytes as evidenced by their larger nuclei size (Figure 2e), and also likely in Kupffer cells. There was also a trend ( $>$ twofold, albeit nonsignificant) for higher basal intrahepatic TNF and IL-6 mRNA levels that correlated with significantly more $\mathrm{p} 65^{+}$nuclei/high power field (mostly hepatocytes) in HET versus WT livers (Figure 2e). These results suggest that HET livers suffer subtle baseline inflammation and NF- $\kappa$ B activation, predominating in hepatocytes, that amplifies following $\mathrm{PH}$. However, A20 heterozygocity did not increase basal nor early post-PH apoptosis of hepatocytes (Figure 2f), indicating that lower A20 levels in these mice were still sufficient to prevent excessive hepatocyte apoptosis following $\mathrm{PH}$.

A20 HET alters expression levels of the cell cycle regulators p21 and cyclins D1, E and $A$ to negatively impact hepatocyte proliferation. Following priming, hepatocyte progression through cell cycle requires intrahepatic production of growth factors, that is, hepatocyte growth factor (HGF) and transforming growth factor alpha (TGFa). ${ }^{18} \mathrm{We}$ noted comparable peak of HGF and TGFa mRNA levels in HET and WT livers $1 \mathrm{~d}$ post PH. However, HET livers neither demonstrated the $4 \mathrm{~d}$ HGF mRNA peak, nor the prolonged increase in TGFa mRNA (80-120-fold up to 4 d) noted in WT livers post $\mathrm{PH}$ (Figures $3 \mathrm{a}$ and $\mathrm{b}$ ).

We recently showed that A20 overexpression in mouse livers promotes hepatocyte proliferation by decreasing $p 21$ mRNA and protein levels. ${ }^{4,11}$ We expected the reverse in HET livers, yet baseline and $\mathrm{PH}$-induced upregulation of $\mathrm{p} 21$ mRNA was comparable in HET and WT livers (Figure $3 \mathrm{c}$ ). However, by immunohistochemistry, the number of $\mathrm{p} 21^{+}$ hepatocytes/high power field was significantly higher in HET versus WT livers $2 \mathrm{~d}$ post $\mathrm{PH}$ (Figure $3 \mathrm{~d}$ ), with a trend towards higher number at baseline. We noted a similar pattern by western blot that showed that intra-hepatic p21 protein levels were higher in HET versus WT livers both before and $2 \mathrm{~d}$ post $\mathrm{PH}$ (Figure 3e). To ensure that heightened p21 protein levels in HET livers simply relate to a shift in its circadian cycling, we evaluated p21 protein levels at $8 \mathrm{am}, 12 \mathrm{pm}$ and $4 \mathrm{pm}$. HET livers showed drastically higher p21 protein levels than WT livers, at all time points tested (Figure 3f), which indicated that increased p21 protein levels in HET livers was constant, at 
least in the daytime when $\mathrm{PH}$ were performed. In a timecourse analysis following $\mathrm{PH}$, we showed, as expected, that intra-hepatic levels of p21 do decrease 1d post-PH in HET livers, albeit not to the extent observed in WT livers, and quickly return to their high baseline values within $2 \mathrm{~d}$ post $\mathrm{PH}$ to remain elevated thereafter (Figure $3 \mathrm{~g}$ ).

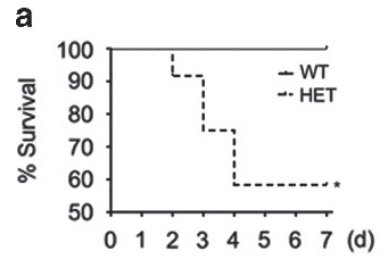

e
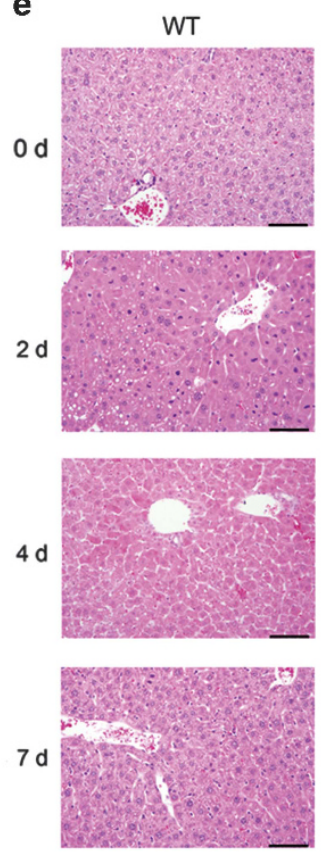

b

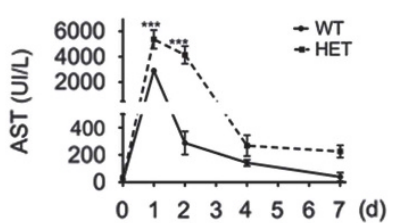

C

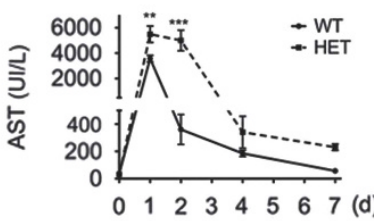

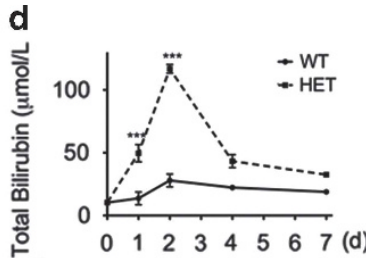

f
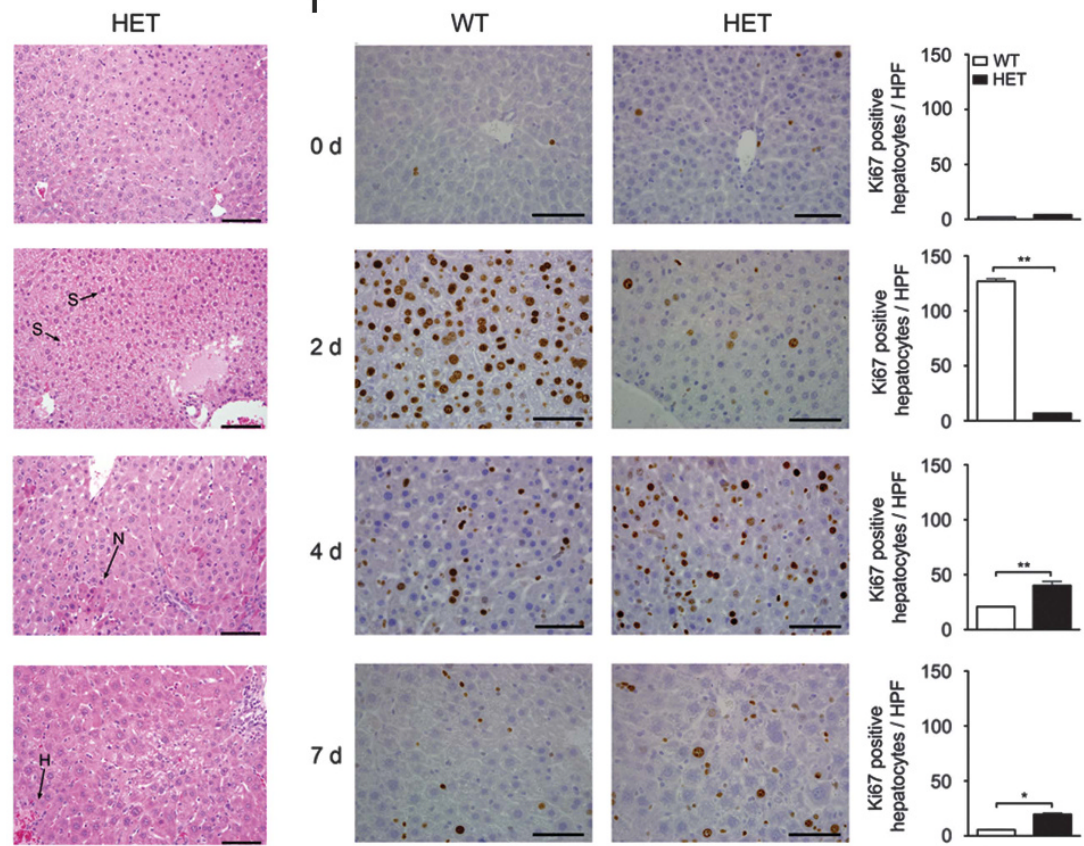

g

$\frac{\text { Oh }}{\text { WT }}$ HET $\mathrm{WT}$ HET

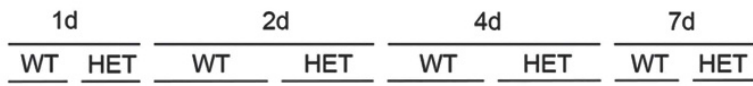
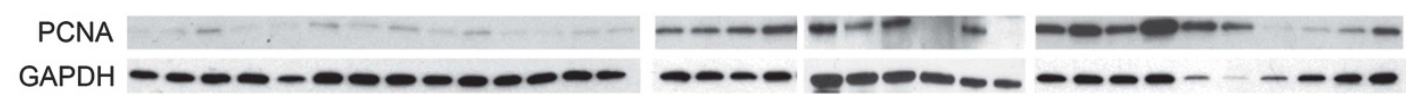

h

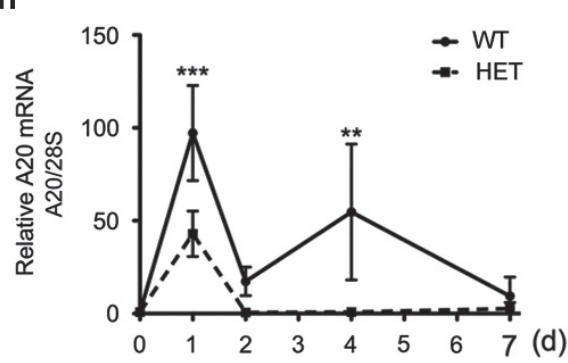

i

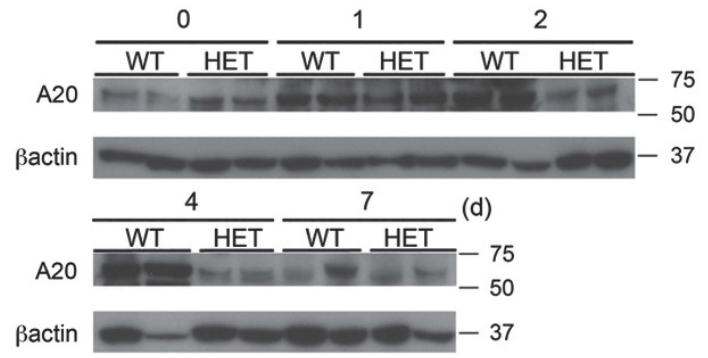

Figure 1 A2O HET provokes lethality by increasing liver damage and impairing hepatocyte proliferation following PH. (a) Kaplan-Meier survival plot compares survival rates following PH in WT and HET mice ( $n=10-14$ mice/group). Plasma and livers from WTand HET mice that survived PH were recovered before (0) and 2, 4 and 7 days (d) after PH an assayed for (b) alanine transaminase (ALT), (c) aspartate transaminase (AST) and (d) total bilirubin. Graphs represent mean \pm S.E.M. of plasma levels ( $n=3-6$ mice/group/ time point). (e) Representative photomicrographs of hematoxylin and eosin and (f) Ki-67 immunostaining (brown). Scale bars: $100 \mu \mathrm{m}$ for (e) and $50 \mu \mathrm{m}$ for (f). Graphs represent mean \pm S.E.M. of Ki67+ cells/high power field (HPF). $\mathrm{S}=$ steatosis, $\mathrm{N}=$ necrosis, $\mathrm{H}=$ hemorrhage. $n=3$ mice/group/time point, ${ }^{*} P<0.05$, ${ }^{* \star} P<0.01$, ${ }^{* * *} P<0.001$. (g) Representative western blots of the cell cycle marker PCNA in WT and HET mouse livers before (0), $4 \mathrm{~h}(\mathrm{~h})$, and 1,2, 4 and 7 days after PH. GAPDH (immunoblots were used as loading control ( $n=2-4$ mice/group/time point). A20 knockdown in HET livers before $(0 \mathrm{~d})$ and 1, 2, 4 and $7 \mathrm{~d}$ after PH was confirmed by (h) qPCR (graphs represent mean \pm S.E.M. of A20/tnfaip3 mRNA levels normalized to $28 \mathrm{~S}$ rRNA levels, and expressed relative to $0 \mathrm{~d}$ WT), and by (i) western blot analysis. $\beta$-actin immunoblots were used as loading control ( $n=2-4$ mice/group/time point) 


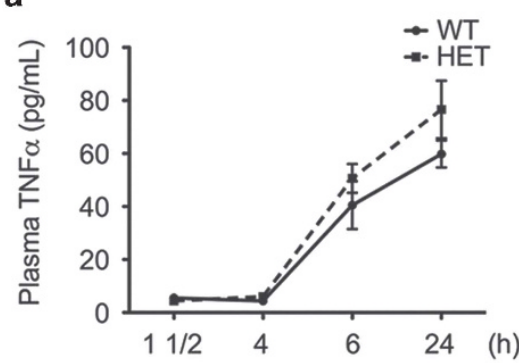

C

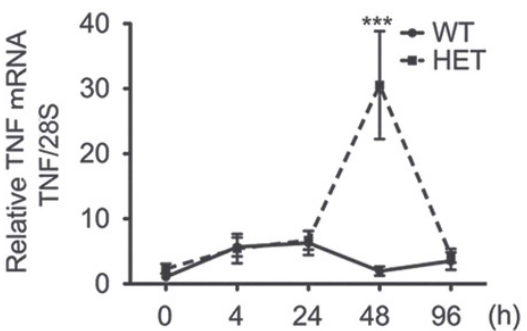

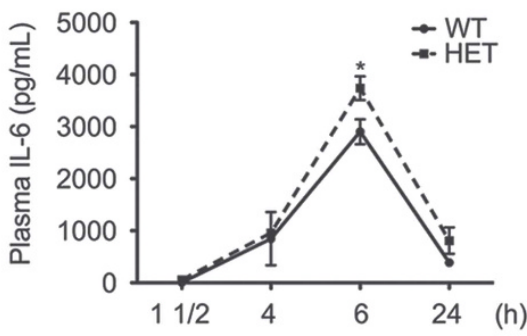

d

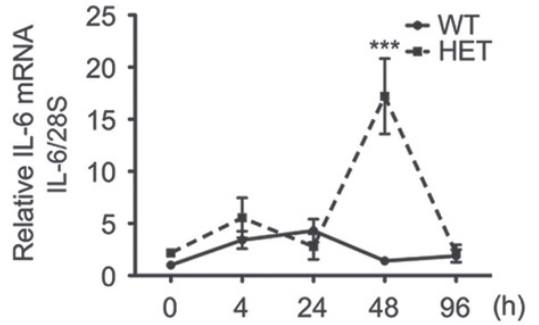

e

WT
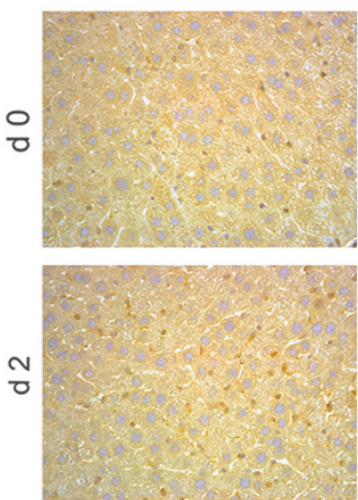

f
HET
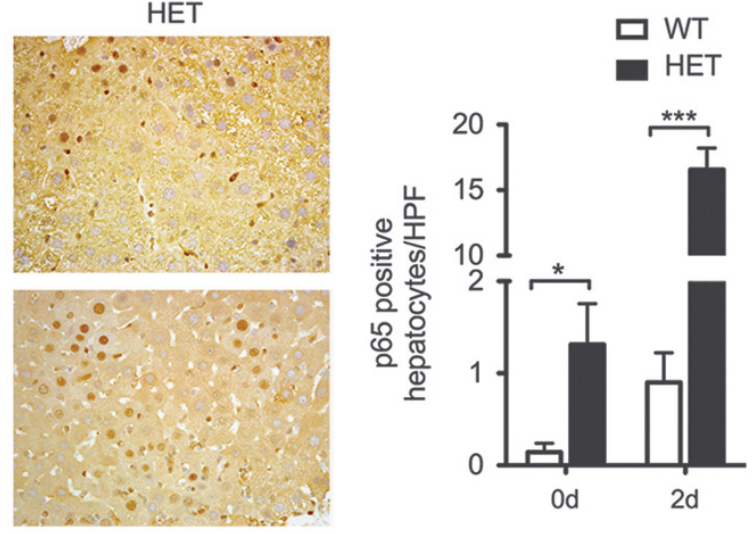

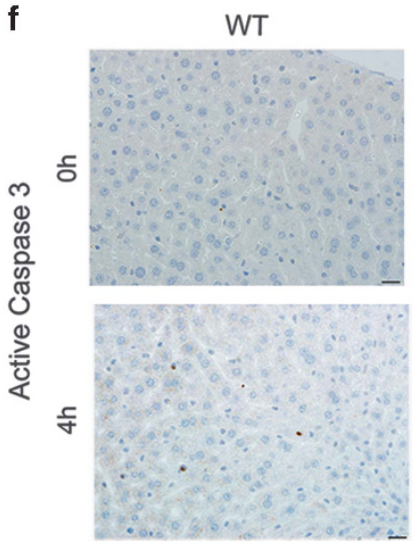

HET

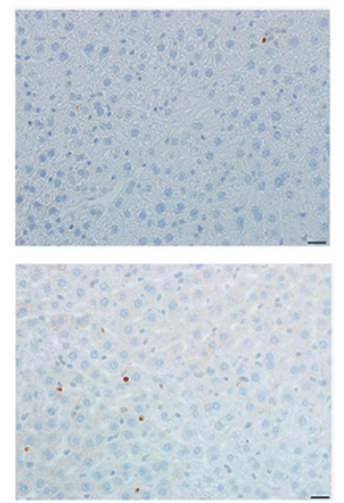

Figure 2 A20 HET mice show higher intrahepatic levels of pro-inflammatory and priming cytokines, IL-6 and TNF, following PH. This correlates with increased activation of $\mathrm{NF}-\kappa \mathrm{B}$ in hepatocytes, but without aggravated apoptosis. Plasma samples were recovered from WTand A20 heterozygous (HET) mice before $(0)$ and at several time points (1 0.5 , 4, $24 \mathrm{~h}$ ) after PH and evaluated by ELISA for plasma levels of (a) TNF and (b) IL-6. Livers from WT and A20 HET mice were recovered before (0) and 4, 24, 48 and $96 \mathrm{~h}$ after PH and evaluated for (c) TNF and (d) IL-6 intrahepatic mRNA levels by qPCR. Graphs represent mean \pm S.E.M. of mRNA levels relative to WTat time 0 , respectively $(n=3-8$ mice per group). $28 \mathrm{~S}$ ribosomal (rRNA) was used as housekeeping gene. (e) Representative photomicrographs of p65 immunostaining (brown) before and $24 \mathrm{~h}$ after PH. Scale bars: $50 \mu \mathrm{m}$. Graph represents mean \pm S.E.M. of $065^{+}$hepatocyte nuclei per high power field (HPF). Only p65 nuclei that were considered hepatocytes based on size were counted ( $n=3$ mice/group). ${ }^{*} P<0.05$, ${ }^{* * *} P<0.001$. (f) Representative photomicrographs of active caspase 3 immunostaining (brown) before and $24 \mathrm{~h}$ after $\mathrm{PH}$. Scale bars: $50 \mu \mathrm{m}$ 

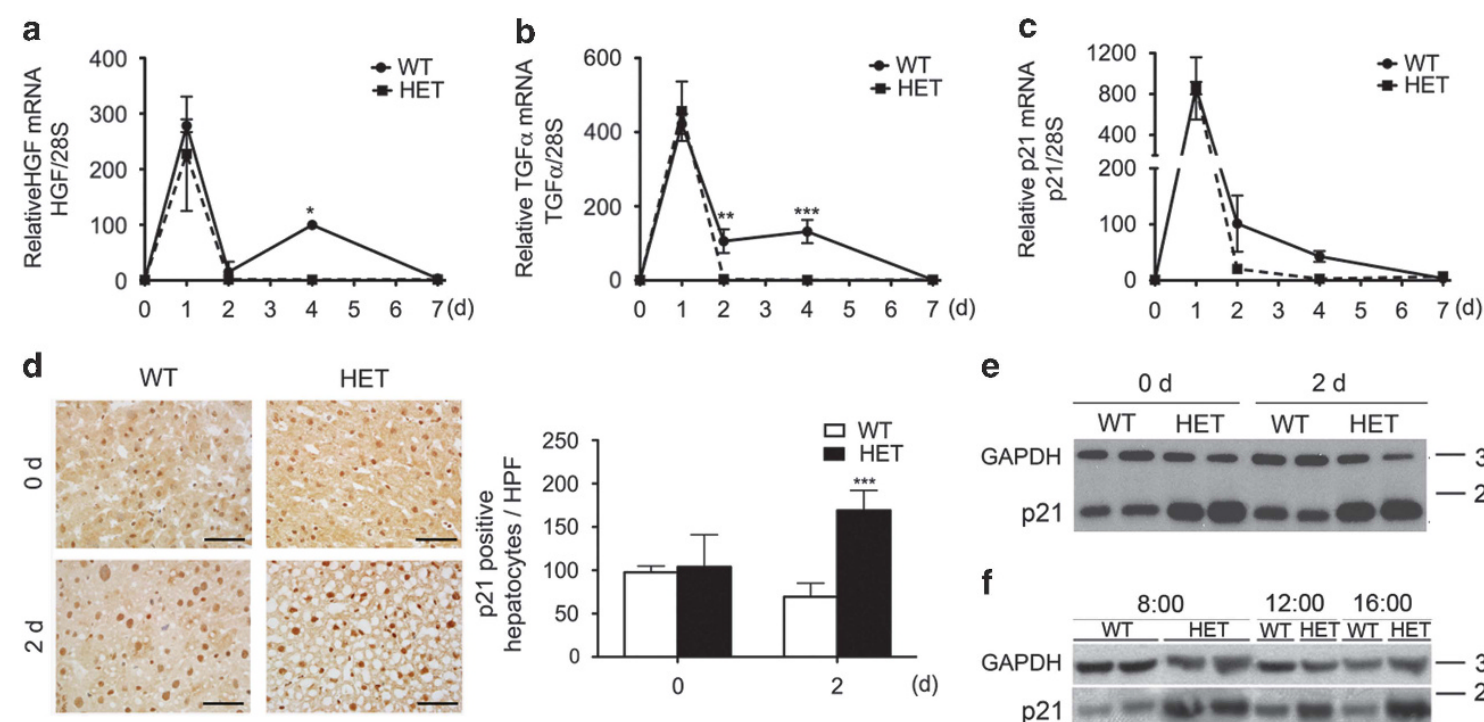

HET

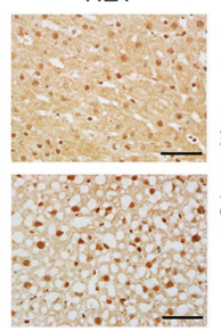

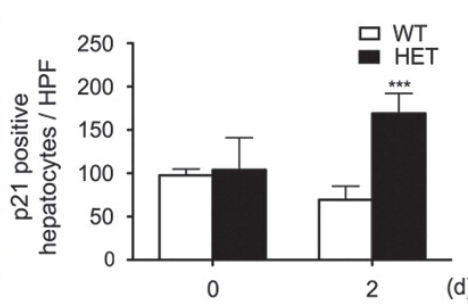

e

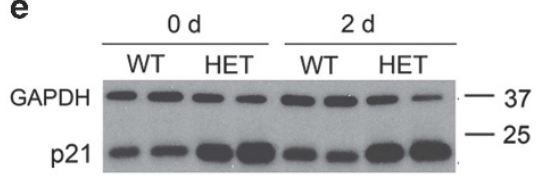

f
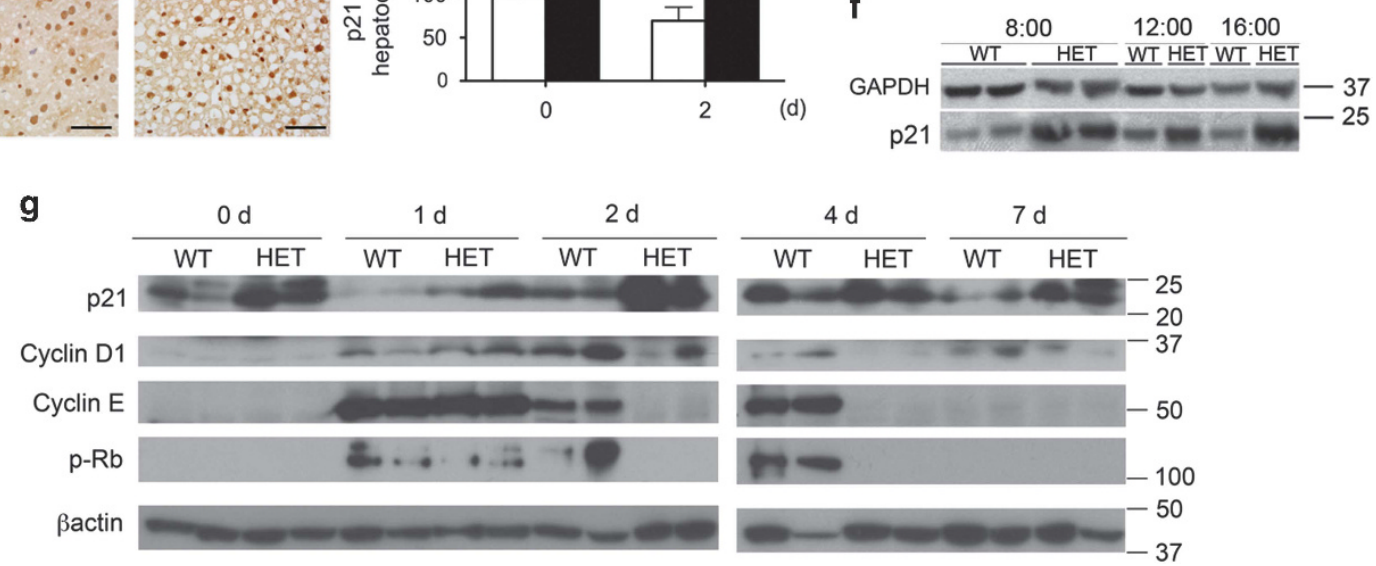

Figure 3 A20 HET negatively modulates hepatic expression of cell cycle regulators before and after PH. Livers from WT and A20 heterozygous (HET) mice were recovered before (0) and 1, 2, 4 and $7 \mathrm{~d}$ after PH and evaluated for intrahepatic mRNA levels of (a) HGF, (b) TGF $\alpha$ and (c) p21, by qPCR. Graphs represent mean \pm S.E.M. mRNA levels normalized to levels of housekeeping $28 \mathrm{~S}$ rRNA, expressed relative to 0 WT mRNA levels ( $n=3-4$ mice/group/time point). (d) Representative photomicrographs of nuclear p21 protein expression by immunohistochemistry (brown) before and $2 \mathrm{~d}$ after PH. Scale bars: $50 \mu \mathrm{m}$. Graph represents mean \pm S.E.M. of p21 ${ }^{+}$nuclei per high power field (HPF) ( $n=3$ mice/group). (e) Representative western blots of p21 in WT and HET mouse livers before ( $0 \mathrm{~d})$ and $2 \mathrm{~d}$ after PH and, (f) at different time points during the diurnal cycle, ranging from $8.00 \mathrm{am}$ to $16.00 \mathrm{pm}$. (g) Representative western blots of p21, cyclin D1, cyclin E and phosphorylated retinoblastoma $(P$-Rb) in WT and HET mouse livers before $(0 \mathrm{~d})$ and 1, 2, 4 and $7 \mathrm{~d}$ after PH. GAPDH (e, f) and $\beta$-actin (g) immunoblots were used as loading control ( $n=2-3$ mice/group/time point). ${ }^{*} P<0.05,{ }^{* \star} P<0.01,{ }^{* * \star} P<0.001$

Prolonged upregulation of cyclin D1, a rate-limiting cell cycle protein, after $\mathrm{PH}$ is required for adequate $\mathrm{LR} .{ }^{19}$ We noted transient increase of the cyclin D1 protein $1 \mathrm{~d}$ post PH in HET livers contrasting with higher ( $2 \mathrm{~d}$ peak) and prolonged (up to 7 d) induction in WT livers (Figure 3f). Similarly, upregulation of cyclin $\mathrm{E}$ and phosphorylation of retinoblastoma protein $(\mathrm{Rb})$ post $\mathrm{PH}$ were transient (only $1 \mathrm{~d}$ ) in HET, while prolonged (up to $4 \mathrm{~d}$ ) in WT livers (Figure 3f). Cyclin A protein levels failed to increase 2 and $4 \mathrm{~d}$ following PH in HET livers, as they did in WT livers (Supplementary Figure 1), but did increase in a delayed fashion (7 d) in HET mice that survived PH. Altogether these data indicate that hepatocyte proliferation post $\mathrm{PH}$ is aborted and/or delayed in A20 HET livers.

Minimal baseline differences in HET liver transcriptome
transform into significant changes following PH. To
decipher the molecular basis for defective LR in HET mice,
we compared HET versus WT liver transcriptomes by
microarray analysis. We identified 90 and 1081 significantly
differentially expressed transcripts pre- and post PH, respec-
tively (Supplementary Tables I and II), and established a
hierarchy of the top 25 and top 50 pre- and post-PH genes Cell Death and Differentiation
(Supplementary Figures 2a and b). By gene ontology (GO) and interactive network enrichment analyses using DAVID, we identified circadian rhythm (CR) as the only significantly affected GO category in HET livers at baseline (Figure 4a). Differentially expressed CR genes included the key transcription regulator of cyclic genes $D$ site of albumin promoterbinding protein $(D B P)^{20}$ that was higher in HET versus WT livers. Other differentially expressed cyclic genes pre-PH included the regulators of lipid and glucose metabolism regulator of G-coupled receptor signaling 16 (RGS16), pyruvate dehydrogenase kinase isozyme 4 (PDK4) and insulin-induced regulator of SREBP1c (INSIG2). GO categories significantly affected in HET versus WT livers post PH largely related to lipid metabolism (Figure 4a). Differentially expressed genes post PH mostly affected lipid metabolism, cell cycle and inflammation (Supplementary Table III). These results agree with lipid metabolism being the most affected GO category in A20 gain-of-function studies. ${ }^{11}$

We validated differential expression of key pre-PH genes by qPCR and demonstrated significantly higher DBP and RGS16 and lower PDK4 mRNA levels in HET versus WT livers (Figure 4b). However, there was only a trend towards lower

CellDeath and Differentiation 
a
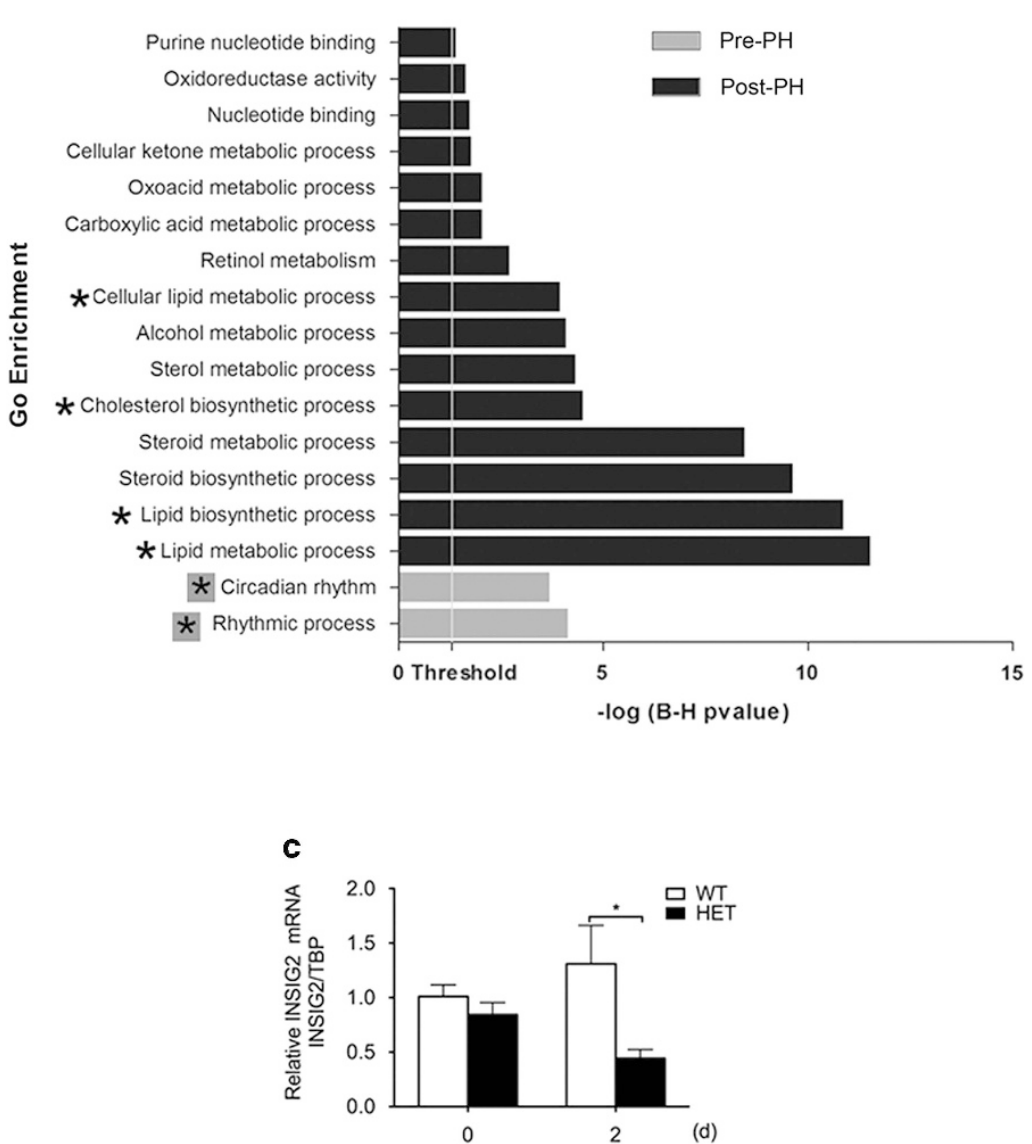
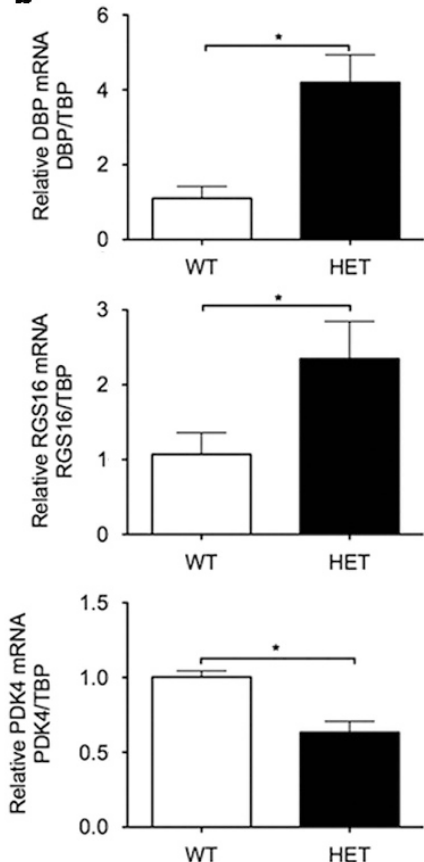

d

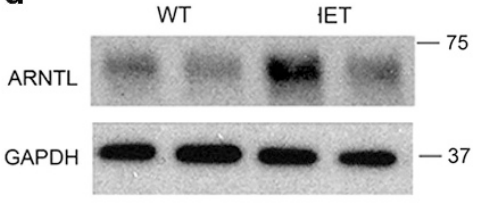

Figure 4 Transcriptional changes induced by A20 knockdown in livers before and after PH identifies CR (before PH) and lipid metabolism (after PH) as top differentially affected GO categories. (a) GO enrichment analysis of differentially expressed genes pre- (grey bars) and $24 \mathrm{~h}$ post PH (Black bars). GO categories with Benjamini-Hochberg corrected $P$-value $<0.05$ were considered significant. GO categories relating to $\mathrm{CR}$ (grey boxed asterix) and lipid metabolism (black asterix) were at the top differentially affected GO categories, pre- and post PH, respectively. (b) We confirmed by qPCR signficant differences in hepatic mRNA levels of liver-specific circadian transcription factor DBP, and of the cyclic regulators of lipid and glucose metabolism RGS16, and PDK4 in HET versus WT mice pre-PH. (c) Although hepatic mRNA levels of the cyclic INSIG2 only showed a trend towards lower levels in HET versus WT livers pre-PH, this trend became significant post PH. Graphs in (b) and (c) represent relative mRNA levels of DBP, RGS16, PDK4 and INSIG2 normalized by mRNA levels of the housekeeping gene TATA-Box binding protein (TBP), and expressed relative to baseline WT mRNA levels. Results are expressed as mean \pm S.E.M. of three mice per group. ${ }^{*} P<0.05$. (d) Upstream of $D P B$, representative western blots of ARNTL protein levels demonstrates higher basal levels of this $C R$ transcription factor in HET versus WT livers. GAPDH immunoblots were used as loading control

INSIG2 mRNA in HET versus WT livers pre-PH, which became statistically significant post $\mathrm{PH}$ (Figure 4c). Upstream of $D B P$, basal intra-hepatic protein (but not mRNA, data not shown) levels of its transcriptional regulator aryl hydrocarbon receptor nuclear translocator like $(A R N T L)$, also referred to as brain and muscle ARNTL (BMAL1), ${ }^{21}$ were substantially higher in HET versus WT livers (Figure $4 d$ ), suggesting that increased $D B P$ mRNA in HET livers was due to its increased transcription.

Impaired lipid metabolism following PH in HET livers results in maladaptive hepatic macrosteatosis. There is ample evidence that $\mathrm{PH}$ prompts the release of FA from adipose tissue and their uptake by the liver, promoting the accumulation of triglycerides (TG) to provide the energy required to fuel $L R .^{22,23}$ Suppression of fat accumulation in the liver after $\mathrm{PH}$ impairs LR in mice. ${ }^{24}$ On the other hand, excessive liver steatosis, such as in leptin- and leptin- receptor-deficient mice ${ }^{25-27}$ or high fat diet-fed mice, ${ }^{28}$ also derails LR post $\mathrm{PH}$. At the molecular level, ob/ob and high fat diet-fed mice show decreased expression of cyclin D1, and heightened p21 protein levels, ${ }^{29}$ which recapitulates the molecular signature we identified in HET mice. Because limited and rather subtle differences in pre-PH HET liver transcriptome significantly impact genes involved in lipid metabolism post $\mathrm{PH}$, we questioned whether defective LR in these mice correlated with inadequate fat redistribution and accumulation in the liver. We confirmed that HET livers suffer severe macrosteatosis, as evidenced by oil red-O staining (Figure 5a), and accumulate higher levels of intrahepatic TG than WT livers following $\mathrm{PH}$ (Figure $5 \mathrm{~b}$ ). The maladaptive hepatic macrosteatosis and TG accumulation that develop post $\mathrm{PH}$ in HET livers evoke similar pathologic features of diabetic and obese mice, strongly suggesting that they are central to the pathogenesis of impaired LR in these mice. 
a

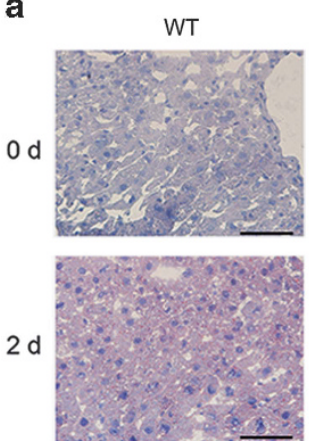

HET

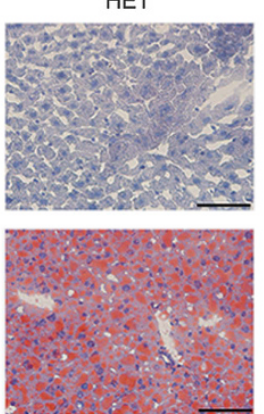

b

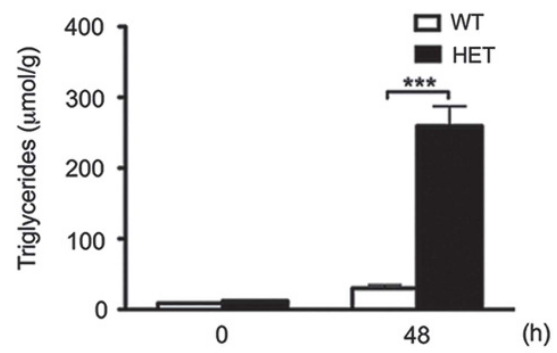

Figure 5 A20 HET associates with maladaptive hepatic macrosteatosis, and excessive intra-hepatic TG levels following PH. (a) Representative photomicrographs of Oil-red $O$ staining showing lipid deposition in HET livers before $(0 \mathrm{~d})$ and $2 \mathrm{~d}$ after $\mathrm{PH}(n=3$ mice/group/time point). Scale bar $=100 \mu$ m. (b) Graph shows quantification of intrahepatic TG levels by gas chromatography before (0) and $2 \mathrm{~d}$ after PH. Results are expressed as $\mu \mathrm{mol}$ of TGs per gram (g) of tissue and shown as mean \pm S.E.M. ( $n=3$ mice/group/time point). ${ }^{* *} P<0.001$

\section{Discussion}

Through loss-of-function studies, we demonstrate that adequate upregulation of $\mathrm{A} 20$ following $\mathrm{PH}$ is essential for appropriate LR. Mere A20 heterozygous knockdown transformed $\mathrm{PH}$ from a well-tolerated procedure to one with more than $40 \%$ lethality, uncovering an otherwise unsuspected phenotype in HET mice. Remarkably, lethality in HET mice topped that of IL-6 KO, ${ }^{30}$ TNF-receptor- $1 \mathrm{KO}^{31}$ and conditional hepatocyte-specific STAT3 $\mathrm{KO},{ }^{32}$ qualifying A20 among the most potent key physiologic regulators of LR.

HET mice that survived PH showed heightened liver damage, macrosteatosis and delayed hepatocyte proliferation, predicting worse results in mice that died. Exploring causes of this drastic outcome, we showed that baseline and first day post-PH surge in circulating and intrahepatic 'priming' yet pro-inflammatory cytokines, TNF and IL-6, were comparable in HET and WT mice. This suggests that A20 HET does not result in hyperactivation of the innate or adaptive immune system as homozygous KO does. ${ }^{13,33}$ However, intrahepatic levels of TNF and IL-6 following PH were excessively higher and prolonged in HET versus WT livers $4 \mathrm{~d}$ post $\mathrm{PH}$, likely contributing to derailed LR. ${ }^{17}$ This indicates that maladaptive inflammatory responses in HET mice only unveil following injury (in this case $\mathrm{PH}$ ), and remain contained to the site of injury (here the liver) as systemic inflammation did not worsen. Our results convincingly show that subtle basal inflammation, as indicated by increased number of $\mathrm{p}^{2} 5^{+}$nuclei in HET livers, mostly corresponding with large nucleolated nuclei of hepatocytes, ${ }^{34}$ significantly aggravates following $\mathrm{PH}$. These results agree with our published data showing that hepatocytes retrieved from A20 HET mice produce higher levels of TNF and IL-6 in response to LPS, than WT hepatocytes. ${ }^{12}$ Excessive and extended production of TNF and IL-6 in HET livers likely relates to their inability to mount an adequate post$\mathrm{PH}$ surge of NF- $\kappa \mathrm{B}$ inhibitory $\mathrm{A} 20$, a pre-requisite for secondary containment of these largely NF-KB-dependent cytokines. ${ }^{13}$ However, even if reduced, levels of the antiapoptotic $\mathrm{A} 20^{9}$ in HET livers following PH were still sufficient to preclude higher rate of hepatocyte apoptosis. This suggests that other anti-apoptotic mechanisms must compensate for lower A20 levels in protecting hepatocytes post $\mathrm{PH}$.
It is well established that IL-6, produced by Kupffer cells and hepatocytes, ${ }^{35}$ is the central trigger of LR via phosphorylation/ activation of STAT3. ${ }^{36}$ However, excessive levels of IL-6 following $\mathrm{PH}$ halt hepatocyte proliferation ${ }^{17}$ by increasing $p 21$ transcription to block Cyclin/CDK complexes ${ }^{37}$ and by binding STAT3 to inhibit its pro-regenerative signals. ${ }^{38}$ Having reported that A20 overexpression decreases p21 mRNA and protein levels, ${ }^{4,11,12}$ we were intrigued by the comparable levels of p21 mRNA in HET and WT livers post PH. This suggested that $\mathrm{A} 20$ was either not a physiologic regulator of p21 mRNA, or that homozygous (not heterozygous) KO of A20 (paralleled by even higher IL-6 levels) was required to increase p21 mRNA levels. Our data showing significantly higher p21 mRNA levels in A20 KO versus WT livers at baseline support the latter, even though it does not indicate whether this regulation is direct or indirect, and whether it is transcriptional or results from changes in mRNA half-life and stability (Supplementary Figure 3). Despite similar mRNA levels, HET mice still demonstrated significantly heightened p21 protein levels at baseline. Also, compared with wipeout of the p21 protein in WT livers, p21 protein levels only moderately and transiently decreased (i.e., in a way that was not conducive for LR) in HET livers. The mechanism(s) supporting heightened p21 levels in A20-deficient mice remain unknown but could relate to the regulation of its ubiquitination and degradation in the proteasome. This would be consistent with our data showing (i) that lower p21 protein levels in A20overexpressing HepG2 hepatoma cells are restored upon treatment of the cells with the proteasome inhibitor MG132, and (ii) that p21 is enriched in MG132-treated HEPG2 protein extracts immunoprecipitated with an anti-ubiquitin antibody (Supplementary Figure 4). Regardless of mechanism(s), our loss-of-function studies suggest that A20 is either directly or indirectly a physiologic transcriptional and posttranscriptional/ posttranslational regulator of $\mathrm{p} 21$. They also expose $\mathrm{p} 21$ as a key culprit in defective LR of HET mice. Transient p21mediated blockade of cell cycle was also suggested as 'the' pathogenic effector of defective LR after extended hepatectomy $(86 \%)^{39}$ and in fatty livers. ${ }^{29}$ This infers that heterozygous A20 KO transforms the molecular signature of a benign $\mathrm{PH}$ to that of either an extended hepatectomy or of a $\mathrm{PH}$ on a marginal fatty liver, plaguing it with equally as high lethality. 
Hepatocyte proliferation following $\mathrm{PH}$ also requires upregulation of HGF and TGFa to induce phase 1 cyclins. ${ }^{18}$ Although A20 HET did not affect early upregulation of HGF and TGFa and consequently that of cyclins $\mathrm{D} 1$ and $\mathrm{E}$ following $\mathrm{PH}$, it blunted their second peak and extended production, precluding prolonged expression of cyclins D1 and E. This pattern of aborted growth factors/cyclins upregulation after an adequate post-PH start in HET mice differs from IL-6 hyperstimulation where their induction post $\mathrm{PH}$ is delayed and their levels lower. $^{17}$

To explore the molecular basis for aborted LR in HET mice, we probed the liver transcriptome and discovered that $\mathrm{A} 20$ HET subtly, but consistently, increased mRNA levels of the CR transcription factor (CRTF) $D B P .{ }^{20}$ Though subtle, this could be quite significant as $5-9 \%$ of the liver transcriptome and $>20 \%$ of its secreted proteome are under CR control. ${ }^{40,41} \mathrm{We}$ traced back higher DBP mRNA levels to higher protein (not mRNA) levels of its transcriptional regulator ARNTL in HET livers. This latter result suggests that hepatic ARNTL is directly or indirectly regulated at the posttranslational level by ubiquitin-editing A20, which would agree with ARNTL incurring posttranslational sumoylation and ubiquitination, ${ }^{42}$ and its protein levels being modulated by proteasomal activity. ${ }^{43}$ Increased ARNTL levels in HET livers correlated with a significant increase in mRNA levels of its other target gene Wee1 (Supplementary Figure 5), a cyclic kinase that inactivates cdc2 to preclude mitosis. ${ }^{44}$ Because ARNTL/ BMAL1 KO mice experience defective hepatocyte proliferation due to increased p21 transcription, ${ }^{45}$ we anticipated that higher ARNTL/BMAL1 levels in HET livers might cause the opposite, that is, decrease p21 transcription. However, p21 mRNA levels remained unchanged with p21 protein levels being significantly higher in HET versus WT livers. This indicates that increased ARNTL levels were not sufficient to impact $p 21$ transcription, and that increased p21 protein levels in HET livers likely relates to ARNTL-independent posttranslational modifications.

Dysregulation of CR also disrupts lipid homeostasis, ${ }^{46,47}$ that is, LR fuel. Specifically, $D B P$ is the dominant regulator of bile acid synthesis rate-limiting enzyme, cholesterol 7 alpha hydroxylase (Cyp7a1). Cyp7a1 levels must rapidly decrease post $\mathrm{PH}$, otherwise bile acids accumulate and cause hepatocyte apoptosis. ${ }^{48}$ Paralleling DPB, Cyp7a1 mRNA levels were higher in HET livers pre- and post $\mathrm{PH}$ (Supplementary Figure 6), confirming higher levels of a functional DBP protein in HET livers. DBP also influences lipid metabolism by inducing insulin-like growth factor binding protein 1 (IGFBP1), which downregulates INSIG2, an inhibitor of de novo lipogenesis. ${ }^{49}$ Higher DBP levels in HET livers coincided with lower pre- and post-PH INSIG2 mRNA levels. As in INSIG2a KO mice, ${ }^{50}$ lower INSIG2 in HET livers associated with deleterious macrosteatosis following $\mathrm{PH}$, hampering LR. ${ }^{27}$

Metabolic genes PDK4 and RGS16 were two other differentially expressed cyclic genes in HET versus WT livers at baseline. PDK4, required for blood glucose homeostasis and ATP production, ${ }^{51}$ was lower in HET versus WT livers. Although lower PDK4 improves diabetes, ${ }^{51}$ it impairs FA oxidation and decreases ATP production, which would hinder LR. RGS16, a regulator of G-protein coupled receptor pathways was higher in HET versus WT livers. Akin A20 HET, hepatocyte-specific overexpression of RGS16 also increases intrahepatic TG levels and provokes regenerationdefective fatty livers. ${ }^{52}$

Altogether, our results establish A20 as an integral physiologic regulator of LR by exposing that mere A20 haploinsufficiency causes defective LR through the additive/ amplifying contribution of heightened p21 protein levels and deregulated lipid metabolism. Recently discovered single nucleotide polymorphisms (SNPs) in the A20/TNFAIP3 locus that correspond with numerous autoimmune and inflammatory diseases highlight the clinical implication of these data. ${ }^{53}$ Indeed, at least five of the A20 SNPs associate with lower A20 expression or activity in homozygous or heterozygous carriers of minor alleles, and hence could recap A20 HET. ${ }^{54-59}$ On the basis of HapMap (Haplotype mapping) data, minor allele frequency of some of these SNPs is far from being negligible, especially in certain populations. For instance, the percentage of homozygous carriers of rs610604 minor allele (prevalent in patients with psoriasis and predicting higher coronary artery disease in diabetic patients) reaches 18\% in Europeans and $32-50 \%$ in Africans, while the percentage of homozygous carriers of rs661561minor allele (associated with Grave's disease) reaches $37 \%$ in Europeans and up to $50 \%$ in Asians. 60,61 However, and beyond the use of A20 SNPs as predictive marker of $L R$, our results mostly reinforce our pursuit of A20based therapies to promote LR and improve safety of extensive LR for donation or tumor.

\section{Materials and Methods}

Mice and PH model. We conducted two-third (67\%) PH in 8-10-week-old female A20 HET and WT littermate mice. All PH were performed between 09001200 hours. This model is a classic model for the study of LR. Resected (pre-PH) and remnant (post-PH) liver tissue was recovered and either embedded in OCT/ snap-frozen for immunohistochemistry, fixed in formalin for morphological analysis or snap-frozen for RNA and protein extraction. Mice received humane care according to criteria of the Guide for the Care and Use of Laboratory Animals. All procedures were approved by the institutional committee for use and care of laboratory animals (IACUC).

Serum collection and measurements of transaminases, bilirubin and cytokines. We drew blood by puncturing the submandibular vein, or catheterizing the vena cava at the time of killing. Serum was recovered and assayed for total bilirubin, aspartate aminotransferase and alanine aminotransferase using commercially available kits (JAS Diagnostics, Inc., Miami Lakes, FL, USA). TNF and IL-6 serum concentration were measured using ELISA Ready-SET-Go! from eBioscience (San Diego, CA, USA).

Histology and immunohistochemistry. For morphological studies, we stained $5-\mu \mathrm{m}$ sections of formalin-fixed liver tissue with hematoxylin and eosin. For immunohistochemistry, frozen sections were incubated with rabbit polyclonal antibodies to mouse p21 (LifeSpan Biosciences Inc., Seattle, WA, USA), cell proliferation marker Ki67 (Dako, Glostrup, Denmark), and NF- $\kappa B$ p65 (Santa Cruz Biotechnology Inc., Santa Cruz, CA, USA), followed by appropriate biotinylated secondary antibodies (Vector, Burlingame, CA, USA). Two investigators blindly evaluated hematoxylin and eosin-stained sections and counted the number of p21, Ki67 and p65-positive hepatocytes per high power field using either automated or manual counting on Image J1.41 (NIH, Bethesda, MD, USA).

Western blot analysis. Whole proteins extracted from liver lysates were analyzed by western blots. Membranes were immunoblotted with mouse monoclonal anti-p21 (BD Biosciences, San Jose, CA, USA), anti-Cyclin-D1 and anti- $\beta$-actin (Santa Cruz); rabbit polyclonal anti-Cyclin E (Millipore, Billerica, MA, USA), anti-Ser780 phospho-Rb (Cell Signaling Technology, Inc., Danvers, MA 
USA,); goat polyclonal anti-p53 (Santa Cruz); and anti-ARNTL/BMAL1 (Novus Biologicals LLC, Littleton, CO, USA), chicken anti-TNFAIP3 (A20) (Abcam Inc., Cambridge, MA, USA), and GAPDH (Calbiochem/EMD Biosciences, Gibston, NJ, USA), followed by appropriate secondary HRP-conjugated antibodies (Thermo Scientific, Rockford, IL, USA). Immunoblots were scanned and band intensity quantified by densitometry using ImageJ 1.41 .

Quantitative reverse transcriptase polymerase chain reaction (qPCR). Whole-liver RNA was extracted using TRIzol (Sigma-Aldrich CO., Saint Louis, MO, USA). Complementary DNA was synthesized using iScript cDNA synthesis kit (Bio-Rad, Hercules, CA, USA). Gene expression was quantified using iTaq Fast SYBR Green Supermix with ROX (Bio-Rad) and gene specific primers (Invitrogen, Coralville, IA, USA) (Supplementary Table IV), or TaqMan Mm00627280_m1 (TNFAIP3), Mm00607939_s1 (Bactin). Expression of target genes was normalized to that of housekeeping genes $\beta$ actin, or TATA box-binding protein (TBP), and quantified using Livak's relative quantification method.

Microarray data analysis. For transcriptional profiling, we analyzed livers from three mice per group, using mouse Affymetrix genome HT 4302.0 PM GeneChip array plate. Quality of scanned arrays images was determined using arrayQualityMetrics package from Bioconductor (http://www.bioconductor.org). Scanned high quality array images were normalized using multichip average algorithm. Genes with $90 \%$ lower confidence bound of the fold change $>1.5$ were considered differentially expressed. To reduce false-positive rate, we preprocessed data by removing the transcripts with lower expression, that is, absolute expression of $<20$ in $90 \%$ of arrays.

GO enrichment analysis. To identify overrepresented GO categories among differentially expressed genes, we used the Biological Processes and Molecular functions Enrichment Analysis available from the Database for Annotation, Visualization and Integrated Discovery (DAVID). A $P$-value gets assigned on the basis of enrichment. GO categories with multiple test corrected $P$-values of $<0.05$ (Holm-Bonferroni method) were considered significant.

Interactive network analysis. We used Ingenuity Pathway Analysis to identify key interaction networks significantly affected in genes differentially expressed in HET versus WT livers pre- and post PH (http//www.ingenuity.com). Ingenuity Pathway Analysis displays the results as score $(-\log p)$ indicating the likelihood of a gene to randomly be in a network or pathway. Ranking networks based on their relevance to the queried data set allows for prioritization of networks with highest impact on the disease process.

Intrahepatic TG content analysis. Total lipid content of liver samples was extracted using the Folch method. ${ }^{62}$ TGs were then isolated by aminopropryl columns (Sigma), and their profiles determined by gas chromatography with a Hewlett Packard 5890 ॥| (Hewlett-Packard, Wilmington, DE, USA), using supercowax-10, $0.25 \mathrm{~mm}$ ID column (Sigma) and a three-stage $\left(150-260^{\circ} \mathrm{C}\right.$ ) temperature program. FA methyl ester peaks were identified by comparison of retention times of a standard mixture, quantified using internal standards, and FA profiles of samples were calculated. Liver's TG content was calculated $(\mu \mathrm{mol} / \mathrm{mg})$ based on TG profiles.

Statistical analysis. We analyzed survival rate by the Kaplan-Meier curve. $P$-values were determined with a log-rank Mantel-Cox test. We used t-test for unpaired two-group comparisons and one- or two-way ANOVA followed by Tukey's and Bonferroni's post hoc test for multiple comparisons. All statistical analyses were done using Prism Mac version 5 (GraphPad Software, La Jolla, CA, USA). Differences between groups were rated significant at a probability error $(P)<0.05$.

\section{Conflict of Interest}

The authors declare no conflict of interest.

Acknowledgements. This study was supported NIH grants R01 DK063275, R01 HL080130 and R21 DK091822 to CF. AIM, JJS, CRP and SMD were supported by NIH T32 HL07734. PS was supported by a grant from the National Swiss Foundation. We also wish to thank Dr. Vishva Dixit and Robert Gerard for providing the A20 plasmid and the recombinant $\beta$-galactosidase adenovirus.

\section{Author contributions}

Study design: PS, CGS, CF. Data acquisition and revision: PS, CGS, JRC, EC, JJS, SMS, CRP, MB, Al. M. Material Support: Av. M. Manuscript drafting: PS, CGS, CF. Important intellectual content: DC, DMS.

1. de Jonge J, Kurian S, Shaked A, Reddy KR, Hancock W, Salomon DR et al. Unique early gene expression patterns in human adult-to-adult living donor liver grafts compared to deceased donor grafts. Am J Transplant 2009; 9: 758-772.

2. Fausto N, Campbell JS, Riehle KJ. Liver regeneration. Hepatology 2006; 43: S45-S53.

3. Dahm F, Georgiev P, Clavien PA. Small-for-size syndrome after partial liver transplantation: definition, mechanisms of disease and clinical implications. Am J Transplant 2005; 5 2605-2610.

4. Longo CR, Patel VI, Shrikhande GV, Scali ST, Csizmadia E, Daniel S et al. A20 protects mice from lethal radical hepatectomy by promoting hepatocyte proliferation via a p21waf1dependent mechanism. Hepatology 2005; 42: 156-164.

5. Van den Eynde M, Hendlisz A. Treatment of colorectal liver metastases: a review. Rev Recent Clin Trials 2009; 4: 56-62.

6. Wertheim JA, Petrowsky H, Saab S, Kupiec-Weglinski JW, Busuttil RW. Major challenges limiting liver transplantation in the United States. Am J Transplant 2011; 11: 1773-1784.

7. Cooper JT, Stroka DM, Brostjan C, Palmetshofer A, Bach FH, Ferran C. A20 blocks endothelial cell activation through a NF-kappaB-dependent mechanism. J Biol Chem 1996; 271: 18068-18073.

8. Wertz IE, O'Rourke KM, Zhou H, Eby M, Aravind L, Seshagiri S et al. De-ubiquitination and ubiquitin ligase domains of A20 downregulate NF-kappaB signalling. Nature 2004; 430 : 694-699.

9. Arvelo MB, Cooper JT, Longo C, Daniel S, Grey ST, Mahiou J et al. A20 protects mice from D-galactosamine/lipopolysaccharide acute toxic lethal hepatitis. Hepatology 2002; 35 : 535-543.

10. Ramsey HE, Da Silva CG, Longo CR, Csizmadia E, Studer $\mathrm{P}$, Patel VI et al. A20 protects mice from lethal liver ischemia/reperfusion injury by increasing peroxisome proliferatoractivated receptor-alpha expression. Liver Transpl 2009; 15: 1613-1621.

11. Damrauer SM, Studer $P$, da Silva CG, Longo CR, Ramsey HE, Csizmadia E et al. A2O modulates lipid metabolism and energy production to promote liver regeneration. PLoS One 2011; 6: e17715.

12. da Silva CG, Studer P, Skroch M, Mahiou J, Minussi DC, Peterson CR et al. A20 promotes liver regeneration by decreasing SOCS3 expression to enhance IL-6/STAT3 proliferative signals. Hepatology 2013; 57: 2014-2025.

13. Lee EG, Boone DL, Chai S, Libby SL, Chein J, Lodolce JP et al. Failure to regulate TNF-induced NF-kB and cell death responses in A20-deficient mice. Science 2000; 289 2350-2354.

14. Zou Y, Bao Q, Kumar S, Hu M, Wang GY, Dai G. Four waves of hepatocyte proliferation linked with three waves of hepatic fat accumulation during partial hepatectomy-induced liver regeneration. PloS One 2012; 7: e30675.

15. Krikos A, Laherty CD, Dixit VM. Transcriptional activation of the tumor necrosis factor alphainducible zinc finger protein, A20, is mediated by kappa B elements. J Biol Chem 1992; 267: 17971-17976.

16. Bach FH, Hancock WW, Ferran C. Protective genes expressed in endothelial cells: a regulatory response to injury. Immunol Today 1997; 18: 483-486.

17. Wustefeld T, Rakemann T, Kubicka S, Manns MP, Trautwein C. Hyperstimulation with interleukin 6 inhibits cell cycle progression after hepatectomy in mice. Hepatology 2000; 32 : 514-522.

18. Fausto N, Laird AD, Webber EM. Liver regeneration. 2. Role of growth factors and cytokines in hepatic regeneration. FASEB J 1995; 9: 1527-1536.

19. Fu M, Wang C, Li Z, Sakamaki T, Pestell RG. Minireview: Cyclin D1: normal and abnormal functions. Endocrinology 2004; 145: 5439-5447.

20. Schrem H, Klempnauer J, Borlak J. Liver-enriched transcription factors in liver function and development. Part II: the C/EBPs and D site-binding protein in cell cycle control, carcinogenesis, circadian gene regulation, liver regeneration, apoptosis, and liver-specific gene regulation. Pharmacol Rev 2004; 56: 291-330.

21. Schibler U. Circadian rhythms. Liver regeneration clocks on. Science 2003; 302: 234-235.

22. Tijburg LB, Nyathi CB, Meijer GW, Geelen MJ. Biosynthesis and secretion of triacylglycerol in rat liver after partial hepatectomy. Biochem J 1991; 277: 723-728.

23. Farrell GC. Probing Prometheus: fat fueling the fire? Hepatology 2004; 40: 1252-1255.

24. Shteyer E, Liao Y, Muglia LJ, Hruz PW, Rudnick DA. Disruption of hepatic adipogenesis is associated with impaired liver regeneration in mice. Hepatology 2004; 40: 1322-1332.

25. Yang SQ, Lin HZ, Mandal AK, Huang J, Diehl AM. Disrupted signaling and inhibited regeneration in obese mice with fatty livers: implications for nonalcoholic fatty liver disease pathophysiology. Hepatology 2001; 34: 694-706.

26. Yamauchi H, Uetsuka K, Okada T, Nakayama H, Doi K. Impaired liver regeneration after partial hepatectomy in db/db mice. Exp Toxicol Pathol 2003; 54: 281-286.

27. Murata H, Yagi T, Iwagaki H, Ogino T, Sadamori $\mathrm{H}$, Matsukawa $\mathrm{H}$ et al. Mechanism of impaired regeneration of fatty liver in mouse partial hepatectomy model. $J$ Gastroenterol Hepatol 2007; 22: 2173-2180

28. DeAngelis RA, Markiewski MM, Taub R, Lambris JD. A high-fat diet impairs liver regeneration in $\mathrm{C} 57 \mathrm{BL} / 6$ mice through overexpression of the NF-kappaB inhibitor, IkappaBalpha. Hepatology 2005; 42: 1148-1157. 
29. Torbenson M, Yang SQ, Liu HZ, Huang J, Gage W, Diehl AM. STAT-3 overexpression and p21 up-regulation accompany impaired regeneration of fatty livers. Am J Pathol 2002; 161: 155-161.

30. Cressman DE, Greenbaum LE, DeAngelis RA, Ciliberto G, Furth EE, Poli V et al. Liver failure and defective hepatocyte regeneration in interleukin-6-deficient mice. Science 1996; 274 1379-1383.

31. Yamada Y, Webber EM, Kirillova I, Peschon JJ, Fausto N. Analysis of liver regeneration in mice lacking type 1 or type 2 tumor necrosis factor receptor: requirement for type 1 but not type 2 receptor. Hepatology 1998; 28: 959-970.

32. Li W, Liang X, Kellendonk C, Poli V, Taub R. STAT3 contributes to the mitogenic response of hepatocytes during liver regeneration. J Biol Chem 2002; 277: 28411-28417.

33. Boone DL, Turer EE, Lee EG, Ahmad RC, Wheeler MT, Tsui $C$ et al. The ubiquitin-modifying enzyme A20 is required for termination of Toll-like receptor responses. Nat Immunol 2004; 5 : 1052-1060.

34. Baratta JL, Ngo A, Lopez B, Kasabwalla N, Longmuir KJ, Robertson RT. Cellular organization of normal mouse liver: a histological, quantitative immunocytochemical, and fine structural analysis. Histochem Cell Biol 2009; 131: 713-726.

35. Panesar N, Tolman K, Mazuski JE. Endotoxin stimulates hepatocyte interleukin-6 production. J Surg Res 1999; 85: 251-258.

36. Li W, Liang X, Kellendonk C, Poli V, Taub R. STAT3 contributes to the mitogenic response of hepatocytes during liver regeneration. J Biol Chem 2002; 277: 28411-28417.

37. Giraud S, Hurlstone A, Avril S, Coqueret O. Implication of BRG1 and cdk9 in the STAT3 mediated activation of the p21waf1 gene. Oncogene 2004; 23: 7391-7398.

38. Coqueret $\mathrm{O}$, Gascan $\mathrm{H}$. Functional interaction of STAT3 transcription factor with the cell cycle inhibitor p21WAF1/CIP1/SDI1. J Biol Chem 2000; 275: 18794-18800.

39. Lehmann K, Tschuor C, Rickenbacher A, Jang JH, Oberkofler CE, Tschopp $O$ et al. Liver failure after extended hepatectomy in mice is mediated by a p21-dependent barrier to liver regeneration. Gastroenterology 2012; 143: 1609-1619 e4.

40. Storch KF, Lipan O, Leykin I, Viswanathan N, Davis FC, Wong WH et al. Extensive and divergent circadian gene expression in liver and heart. Nature 2002; 417: 78-83.

41. Miller BH, McDearmon EL, Panda S, Hayes KR, Zhang J, Andrews JL et al. Circadian and CLOCK-controlled regulation of the mouse transcriptome and cell proliferation. Proc Nat Acad Sci USA 2007; 104: 3342-3347.

42. Lee J, Lee Y, Lee MJ, Park E, Kang SH, Chung $\mathrm{CH}$ et al. Dual modification of BMAL1 by SUMO2/3 and ubiquitin promotes circadian activation of the CLOCK/BMAL1 complex. Mol Cell Biol 2008; 28: 6056-6065.

43. Stratmann M, Suter DM, Molina N, Naef F, Schibler U. Circadian Dbp transcription relies on highly dynamic BMAL1-CLOCK interaction with $\mathrm{E}$ boxes and requires the proteasome. Mol Cell 2012; 48: 277-278.

44. Matsuo T, Yamaguchi S, Mitsui S, Emi A, Shimoda F, Okamura H. Control mechanism of the circadian clock for timing of cell division in vivo. Science 2003; 302: 255-259.

45. Grechez-Cassiau A, Rayet B, Guillaumond F, Teboul M, Delaunay F. The circadian clock component BMAL1 is a critical regulator of $\mathrm{p} 21 \mathrm{WAF} 1 / \mathrm{CIP} 1$ expression and hepatocyte proliferation. J Biol Chem 2008; 283: 4535-4542.
46. Tong X, Yin L. Circadian rhythms in liver physiology and liver diseases. Compr Physiol 2013; 3: $917-940$.

47. Shi SQ, Ansari TS, McGuinness OP, Wasserman DH, Johnson $\mathrm{CH}$. Circadian disruption leads to insulin resistance and obesity. Curr Biol 2013; 23: 372-381.

48. Zhang L, Huang X, Meng Z, Dong B, Shiah S, Moore DD et al. Significance and mechanism of CYP7a1 gene regulation during the acute phase of liver regeneration. Mol Endocrinol 2009; 23: 137-145

49. Yabe D, Brown MS, Goldstein JL. Insig-2, a second endoplasmic reticulum protein that binds SCAP and blocks export of sterol regulatory element-binding proteins. Proc Natl Acad Sci USA 2002; 99: 12753-12758.

50. Yabe D, Komuro R, Liang G, Goldstein JL, Brown MS. Liver-specific mRNA for Insig-2 downregulated by insulin: implications for fatty acid synthesis. Proc Natl Acad Sci USA 2003; 100: 3155-3160.

51. Jeoung $\mathrm{NH}$, Harris RA. Pyruvate dehydrogenase kinase-4 deficiency lowers blood glucose and improves glucose tolerance in diet-induced obese mice. Am J Physiol Endocrinol Metab 2008; 295: E46-E54.

52. Pashkov V, Huang J, Parameswara VK, Kedzierski W, Kurrasch DM, Tall GG et al. Regulator of $G$ protein signaling (RGS16) inhibits hepatic fatty acid oxidation in a carbohydrate response element-binding protein (ChREBP)-dependent manner. J Biol Chem 2011; 286: 15116-15125.

53. Vereecke L, Beyaert R, van Loo G. Genetic relationships between A20/TNFAIP3, chronic inflammation and autoimmune disease. Biochem Soc Trans 2011; 39: 1086-1091.

54. Lodolce JP, Kolodziej LE, Rhee L, Kariuki SN, Franek BS, McGreal NM et al. African-derived genetic polymorphisms in TNFAIP3 mediate risk for autoimmunity. J Immunol 2010; 184: 7001-7009.

55. Wang S, Wen F, Wiley GB, Kinter MT, Gaffney PM. An enhancer element harboring variants associated with systemic lupus erythematosus engages the TNFAIP3 promoter to influence A20 expression. PLoS Genet 2013; 9: e1003750.

56. Boonyasrisawat W, Eberle D, Bacci S, Zhang YY, Nolan D, Gervino EV et al. Tag polymorphisms at the A20 (TNFAIP3) locus are associated with lower gene expression and increased risk of coronary artery disease in type 2 diabetes. Diabetes 2007; 56: 499-505.

57. Thomson W, Barton A, Ke X, Eyre S, Hinks A, Bowes J et al. Rheumatoid arthritis association at 6q23. Nat Genet 2007; 39: 1431-1433.

58. Zhu L, Zhang F, Shen Q, Chen S, Wang X, Wang L et al. Characteristics of A20 gene polymorphisms in T-cell acute lymphocytic leukemia. Hematology 2014; 19: 448-454.

59. Song RH, Yu ZY, Wang Q, Muhali FS, Jiang WJ, Xiao L et al. Polymorphisms of the TNFAIP3 region and Graves' disease. Autoimmunity 2014; 47: 459-465.

60. Frazer KA, Ballinger DG, Cox DR, Hinds DA, Stuve LL, Gibbs RA et al. A second generation human haplotype map of over 3.1 million SNPs. Nature 2007; 449: 851-861.

61. Altshuler DM, Gibbs RA, Peltonen L, Dermitzakis E, Schaffner SF, Yu F et al. Integrating common and rare genetic variation in diverse human populations. Nature 2010; 467: 52-58.

62. Folch J, Lees M, Sloane Stanley GH. A simple method for the isolation and purification of total lipides from animal tissues. J Biol Chem 1957; 226: 497-509.

\section{Supplementary Information accompanies this paper on Cell Death and Differentiation website (http://www.nature.com/cdd)}

